\title{
AVALIAÇÃO DA ATENÇÃO INTEGRAL À SAÚDE DO IDOSO NA PERCEPÇÃO DE PROFISSIONAIS
}

\section{Evaluation of comprehensive health care for older people based on professionals' perceptions}

\section{Evaluación de la atención integral para la salud del mayor desde la percepción de profesionales}

Juliana Fernandes Cabral iD

Universidade do Estado de Mato Grosso - UNEMAT - Tangará da Serra (MT) - Brasil

Jeniffer Fernanda Gonçalves da Silva iD

Universidade do Estado de Mato Grosso - UNEMAT - Tangará da Serra (MT) - Brasil

Josué Souza Gleriano iD

Universidade do Estado de Mato Grosso - UNEMAT - Tangará da Serra (MT) - Brasil

Priscila Balderrama iD

Universidade Federal de Mato Grosso do Sul - UFMS -Três Lagoas (MS) - Brasil

Angélica Pereira Borges (iD)

Universidade do Estado de Mato Grosso - UNEMAT - Tangará da Serra (MT) - Brasil

\section{Ageo Mário Cândido da Silva iD}

Universidade Federal de Mato Grosso - UFMT - Cuiabá (MT) - Brasil

\section{RESUMO}

Objetivo: Avaliar a atenção integral à saúde do idoso segundo a percepção de profissionais em Unidades de Saúde da Família (USF). Métodos: Estudo de abordagem qualitativa, do tipo descritivo-exploratório, realizado no segundo semestre de 2016 em dez Unidades de Saúde da Família (USF) de um município localizado no sudoeste do estado de Mato Grosso, do qual participaram as equipes multiprofissionais das USF. Coletaram-se dados através da técnica de grupo focal. Para análise e interpretação, construíram-se narrativas e submeteu-se à análise de conteúdo do tipo temática. Resultados: O estudo evidenciou que a atenção integral à saúde do idoso na Atenção Primária à Saúde (APS), no município estudado, ocorre de maneira fragmentada e reforça a cultura biomédica. A integralidade, tida como um dos princípios doutrinários do Sistema Único de Saúde (SUS), se encontra fragilizada e pouco valorizada pelos profissionais desse nível de atenção à saúde. Conclusão: Concluiu-se que, no contexto deste estudo, a atenção integral à população idosa ocorre de forma fragmentada e desarticulada. Os profissionais de saúde investigados consideram que o cuidado realizado é puramente clínico e focado em ações curativas ou preventivas.

Descritores: Atenção Primária à Saúde; Saúde do Idoso; Avaliação em Saúde; Gestão em Saúde.

\section{ABSTRACT}

Objective: To evaluate comprehensive health care for older people based on the perceptions of professionals working in Family Health Care Centers (Unidades de Saúde da Familia - USF). Methods: A qualitative descriptive and exploratory study was carried out in the second half of 2016 with multi-professional teams working in ten USF in a municipality located in the southwest of Mato Grosso State. Data were collected using the focus group technique. For analysis and interpretation, narratives were built and submitted to thematic content analysis. Results: The study showed that comprehensive health care for older people in Primary Health Care (PHC) in the municipality analyzed is delivered in a fragmented way and strengthens the biomedical culture. Comprehensiveness, which is acknowledged as one of the doctrinal principles of the Unified Health System (Sistema Único de Saúde - SUS), is weak and undervalued by professionals working at this level of health care. Conclusion: In the context of this study, comprehensive health care for the older population was delivered in a fragmented and disjointed way. The health professionals analyzed believed that the care delivered is purely clinical and focused on curative or preventive actions.

Descriptors: Primary Health Care; Health of the Elderly; Health Evaluation; Health Management. 


\section{RESUMEN}

Objetivo: Evaluar la atención integral para la salud del mayor desde la percepción de profesionales de las Unidades de Salud de la Familia (USF). Métodos: Estudio de abordaje cualitativo del tipo descriptivo-exploratorio realizado en el segundo semestre de 2016 en diez Unidades de Salud de la Familia (USF) de un municipio localizado en el sudoeste del estado de Mato Grosso del cual han participado los equipos multiprofesionales de las USF. Se recogieron datos a través de la técnica de grupo focal. Para el análisis e interpretación se han construido narrativas y se las han analizado por el análisis de contenido del tipo temático. Resultados: El estudio ha evidenciado que la atención integral para la salud del mayor en la Atención Primaria de Salud (APS) en el municipio estudiado se da de manera fragmentada y refuerza la cultura biomédica. La integralidad que es uno de los principios doctrinarios del Sistema Único de Salud (SUS) está fragilizada y poco valorada por los profesionales de ese nivel de atención a la salud. Conclusión: Se concluye que en el contexto de ese estudio la atención integral a la población mayor se da de manera fragmentada y desarticulada. Los profesionales sanitarios investigados consideran que el cuidado realizado es solamente clínico y dirigido para las acciones curativas o de prevención.

Descriptores: Atención Primaria de Salud; Salud del Anciano; Evaluación en Salud; Gestión en Salud.

\section{INTRODUÇÃO}

O Sistema Único de Saúde (SUS), que marca a inclusão social no país e uma afirmação política dos direitos dos cidadãos, fundamenta-se nos princípios doutrinários que conferem universalidade, integralidade e equidade. Constitui-se em uma das maiores conquistas sociais alcançadas com o objetivo de coordenar e integrar as ações de saúde, nas três esferas de governo, para atender demandas assistenciais à saúde, que passam a ser universais e descentralizadas ${ }^{(1)}$.

A reorganização da atenção no SUS, a partir da Atenção Primária à Saúde (APS), tem contribuído, no território brasileiro, para uma aproximação dos objetivos da APS no conjunto de ações, de caráter individual e coletivo, nos eixos de promoção, proteção, prevenção de agravos, diagnóstico, tratamento, reabilitação, redução de danos, cuidados paliativos e vigilância em saúde, desenvolvida através de um cuidado integrado e de gestão qualificada, além de atenção multiprofissional em territórios delimitados ${ }^{(2)}$.

O foco das ações organizadas pelo SUS deve possibilitar desde o primeiro contato do usuário com a rede assistencial até a continuidade, com coordenação da atenção do sistema, no intuito de permitir avanço na integralidade, atributo essencial para o fortalecimento da $\mathrm{APS}^{(3)}$.

Com base neste panorama, evidencia-se um avanço substancial na construção do SUS ao longo dos últimos anos, de modo que a necessidade de avaliação da implementação de iniciativas que reconheçam a qualidade dos serviços ofertados à população, através da execução de ações de monitoramento e avaliação de processos e resultados, torna-se essencial(4).

A cultura de avaliação ainda não se consolidou no Brasil, mas compreende-se que a avaliação é um instrumento fundamental para a gestão na medida em que subsidia a tomada de decisão de maneira mais assertiva( ${ }^{(5)}$ e está presente nas diretrizes políticas, uma vez que a sua utilização é preconizada como recurso para melhoria dos serviços de saúde ${ }^{(6)}$. A sua constituição interna e o uso articulado para a tomada de decisão fortalecem seu reconhecimento e incorporação como relevante instrumento de gestão(7).

Nesse sentido, o Ministério da Saúde (MS), por meio da Portaria n. 1.654, do Gabinete do Ministro, de 19 de julho de 2011, implementou o Programa Nacional de Melhoria do Acesso e da Qualidade da Atenção Básica (PMAQ-AB), que apresenta a Autoavaliação para Melhoria do Acesso e da Qualidade da Atenção Básica (AMAQ) como ferramenta indutora de um processo permanente e progressivo de ampliação do acesso e de qualificação das práticas de gestão, de cuidado e de participação na $\mathrm{APS}^{(8)}$.

O PMAQ tem como objetivo primordial impulsionar a ampliação do acesso e a melhoria da qualidade da atenção básica, com garantia de um padrão de qualidade comparável nacional, regional e localmente, de maneira a permitir maior transparência e efetividade das ações governamentais direcionadas à Atenção Básica em Saúde ${ }^{(9)}$.

A utilização de instrumentos autoavaliativos qualifica os processos na APS, porque possibilita o agir reflexivo ao orientar a ação das equipes. Além disso, os instrumentos autoavaliativos são utilizados como estratégia permanente para tomada de decisão ${ }^{(8)}$. A concepção de atenção integral utilizada pelo PMAQ-AB afirma uma atenção à saúde que envolve o acolhimento da demanda programática e espontânea, de diferentes grupos populacionais, em diferentes fases do desenvolvimento humano, como infância, adolescência, idade adulta e terceira idade ${ }^{(10)}$. 
Nesse contexto, a atenção integral à saúde do idoso assume destaque, visto que tal população protagoniza um processo de ascensão mundial e figura como realidade no nosso país. Atualmente, $11,8 \%$ da população brasileira constituem-se de pessoas com 60 anos ou mais, correspondendo a aproximadamente 23 milhões de pessoas. Projeções estatísticas apontam que, no ano de 2025, o Brasil ocupará o $6^{\circ}$ lugar do mundo em número de idosos ${ }^{(11)}$.

Nesse sentido, este estudo apresenta como objetivo avaliar a atenção integral à saúde do idoso segundo a percepção de profissionais em Unidades de Saúde da Família (USF).

\section{MÉTODOS}

Estudo de abordagem qualitativa, do tipo descritivo-exploratório, realizado no segundo semestre de 2016, em dez Unidades de Saúde da Família (USF) de um município localizado no sudoeste do estado de Mato Grosso, do qual participaram as equipes multiprofissionais das USF.

A adesão do município de Tangará da Serra, no sudoeste do estado de Mato Grosso, ao PMAQ-AB aconteceu em 2016, que corresponde ao terceiro ciclo do PMAQ no Brasil. Esse município, a partir do ano de 2014, acelerou exponencialmente o processo de expansão da atenção básica a partir do Programa Mais Médicos, e tornou-se referência no estado, chegando a $100 \%$ de $\operatorname{cobertura}^{(7)}$.

A Secretaria Municipal de Saúde/Coordenação de Atenção Básica realizou o convite ao Escritório de Qualidade para Organizações de Saúde (EsQualos/UNEMAT), um programa de extensão com interface de pesquisa, para que praticasse com as equipes cadastradas no PMAQ-AB o processo de sensibilização e simulação das fases de avaliação. Assim, desenhou-se uma pesquisa de abordagem qualitativa, do tipo exploratória e descritiva, que integrou um dos eixos de avaliação da pesquisa matricial intitulada "Percepções profissionais para a atenção integral a partir da organização do processo de trabalho em unidades básicas de saúde".

A pesquisa contemplou Unidades de Saúde da Família que estavam cadastradas para receber a visita de avaliação no $3^{\circ}$ ciclo do PMAQ-AB ${ }^{(12)}$. Participaram do estudo dez equipes de saúde da família, totalizando setenta e oito profissionais: enfermeiros, médicos, técnicos de enfermagem, recepcionistas, agentes comunitários de saúde (ACS), cirurgiões-dentista e técnicos de higiene bucal. Adotou-se como critério de exclusão o profissional estar cumprindo afastamento ou férias no dia da reunião agendada.

Coletaram os dados, no segundo semestre de 2016, os pesquisadores responsáveis pelo estudo, utilizando a técnica de grupo focal ${ }^{(13)}$, sendo as entrevistas guiadas pelo instrumento validado pelo Ministério da Saúde, a AMAQ, nas subdimensões Organização do Processo de Trabalho e Atenção Integral à Saúde.

Através da AMAQ avalia-se se os profissionais prestam um atendimento com qualidade, balizando suas respostas por meio da descrição dos padrões de qualidade estabelecidos no instrumento, ou seja, qualidade em saúde é definida como o nível de atendimento a padrões de qualidade previamente estabelecidos, atinentes a normas, protocolos e diretrizes que organizam as ações e as práticas ${ }^{(10)}$.

Os oito itens que contemplam a descrição do padrão de qualidade concernente ao acompanhamento integral da saúde do idoso são: (a) Existência de cadastro atualizado dessa população no território; (b) Realização de atendimento domiciliar aos idosos impossibilitados de se locomover; (c) Monitoramento da cobertura vacinal; (d) Realização de exame da cavidade bucal e da superfície corporal, em todas as consultas médicas e de enfermagem, com a finalidade de identificar lesão cancerosa e maus-tratos; (e) Intervenções para detecção precoce das principais demências que incidem nessa população (Parkinson, Alzheimer etc.); (f) Acompanhamento pela equipe de saúde bucal; (g) Intervenções junto às famílias para identificar e capacitar cuidadores que irão prestar assistência domiciliar adequada; (h) Utilização da caderneta do idoso em todas as situações de atendimento ${ }^{(12)}$.

Cada equipe discutiu e avaliou o grau de adequação das suas práticas ao padrão de qualidade apresentado através de uma escala de pontuação que varia entre 0 e 10 pontos, sendo o ponto 0 (zero) indicativo do não cumprimento do padrão e o ponto 10 a total conformidade. Os intervalos entre 0 e 10 representam os diferentes graus de atendimento da situação analisada em relação à qualidade almejada. Após atribuição da nota, a equipe tinha que justificar o motivo do consenso. Optou-se, então, como método de análise, pelo cálculo de média dos pontos atribuídos por cada equipe ao padrão referente à atenção integral à saúde do idoso ${ }^{(12)}$.

Os encontros audiogravados duraram cerca de quatro horas e, posteriormente, realizou-se a transcrição das falas na íntegra. Para identificar os responsáveis pelas falas e não perder a importância de cada participante, a equipe de pesquisa utilizou um diário de bordo ${ }^{(14)}$ e contou com quatro pesquisadores em cada reunião. Submeteuse o material à leitura exploratória para apropriação da totalidade do conteúdo e, posteriormente, realizou-se leitura exaustiva para apreender em profundidade o teor do material. 
Para análise e interpretação, os pesquisadores construíram narrativas ${ }^{(15)}$. Em seguida, analisaram os núcleos argumentais de cada narrativa, comparando-as entre si, a fim de identificar suas diferenças e semelhanças, submetendo-as à análise de conteúdo, na vertente representacional temática de Bardin ${ }^{(16)}$, alicerçada nas fases de pré-análise, análise e exploração( ${ }^{(17)}$.

O projeto obteve aprovação do Comitê de Ética em Pesquisa da Universidade do Estado de Mato Grosso, expressa por meio do Parecer $n^{\circ}$. 1.385.829.

\section{RESULTADOS E DISCUSSÃO}

Ao compilar as notas atribuídas por cada equipe ao padrão "A equipe de atenção básica realiza acompanhamento integral da saúde do idoso", identificou-se uma média de 7,5 pontos, com mínima de 3,0 pontos e máxima de 9,0 pontos.

Após a análise do material e divisão dos núcleos de sentido, emergiram duas categorias nomeadas como: "Desafios na gestão e organização do processo de trabalho para a atenção integral à saúde do idoso" e "Desafios na implementação da atenção qualificada ao idoso".

\section{Desafios na gestão e organização do processo de trabalho para a atenção integral à saúde do idoso}

A população idosa cresce com maior rapidez do que qualquer outro grupo etário e, diante dessa realidade, tornase irrefutável a adoção de estratégias públicas voltadas para esse grupo, com vistas a otimizar as oportunidades de saúde, de participação e segurança no envelhecimento ${ }^{(11)}$.

Nos relatos abaixo, evidenciam-se alguns aspectos relacionados à gestão e à organização do processo de trabalho que impactam na atenção integral à saúde do idoso, dentre os quais: a utilização da caderneta do idoso, mudanças nos sistemas de informação, acompanhamento da população idosa e realização de atendimento domiciliar aos idosos.

Em relação à caderneta do idoso, os profissionais da atenção básica justificam a não utilização por falta do instrumento nas unidades de saúde:

"A caderneta não está vindo para nós [...] a última caderneta que veio foi em 2014 [...]. Eu acho que o que está faltando é só a caderneta [...] a caderneta nem está vindo para a unidade." (Agente Comunitário de Saúde USF 2)

"Eu não tenho a caderneta do idoso, vou exigir." (Médico USF 4)

"A caderneta do idoso não [...] agora, a utilização da caderneta é pouquíssima, muitos nem conhecem a caderneta." (Médico USF 6)

"[...] agora, quanto à utilização da caderneta, é o que falta." (Médico USF 7)

Observam-se três situações. A primeira é a ausência da caderneta nas unidades. A caderneta é um formulário criado para acompanhamento, tanto do idoso quanto da unidade, e é considerada um documento. A segunda, a pouca utilização das cadernetas de idosos pelos profissionais (daqueles que possuem) e a terceira, a dualidade de utilização enquanto instrumento que é item de um processo de avaliação.

O MS lançou a primeira edição da Caderneta de Saúde do Idoso em 2008 e, atualmente, está em sua quarta edição, publicada em 2017. A nova caderneta recebeu consulta pública e obteve ampla participação de diferentes seguimentos da sociedade, incluindo gestores municipais, estaduais e federais de saúde ${ }^{(18)}$. A caderneta auxilia no bom manejo da saúde da pessoa idosa, sendo usada tanto pelas equipes de saúde quanto pelos idosos, por seus familiares e cuidadores por permitir o registro, bem como o acompanhamento, por um período de cinco anos ${ }^{(19)}$.

Destaca-se, ainda, que as informações necessárias ao seu preenchimento devem ser relatadas pelo próprio idoso, por um familiar ou cuidador, no intuito de se manter a veracidade dos dados obtidos, com posterior elaboração de um plano de cuidado que contemple e atenda a todas as suas necessidades de saúde ${ }^{(18)}$.

Em outra fala, percebeu-se que uma das profissionais de saúde confundiu a Caderneta do HIPERDIA (Sistema de Cadastramento e Acompanhamento de Hipertensos e Diabéticos) com a caderneta do idoso, como se visualiza no trecho a seguir.

"[...] a caderneta sim. As meninas têm uma cadernetinha verdinha que é do HIPERDIA, daí o paciente vem aferir a pressão com a gente a primeira vez, é um idoso que a gente nunca conheceu, e fazemos um mapeamento da sua pressão. As meninas, na hora da visita domiciliar, estão utilizando a caderneta na hora de fazer o cadastro [...]." (Enfermeira USF 10) 
No Mato Grosso, a Caderneta de Saúde da Pessoa Idosa é distribuída somente após a manifestação de interesse dos Secretários Municipais de Saúde, por meio de emissão de ofício ao $\mathrm{MS}^{(20)}$. Nesse contexto, nota-se que a caderneta, apesar de ser um instrumento que serve como guia para a prestação de uma atenção qualificada ao idoso, pode ficar subutilizada em muitos municípios, pelo próprio desconhecimento ou limitações ao acesso( ${ }^{(18)}$.

A carência de insumos e equipamentos necessários constitui-se um fator impeditivo no desenvolvimento de diversas ações e à resolutividade das unidades básicas de saúde, sendo assim limitada sua capacidade de resposta aos problemas de saúde da população(21).

As mudanças nos sistemas de informação também são apontadas como fator interveniente para garantir atenção integral ao idoso. Nas unidades investigadas, os profissionais relatam os seguintes pontos de fragilidades:

\section{"[...] "cada hora é uma tentativa e um sistema de informação diferente [...]." (Enfermeira USF 10) \\ "[...] cadastramos tudo, refizemos um trabalho, ou trabalho dobrado, né, e simplesmente perdeu [...]." (Enfermeira USF 04)}

"[...] não sei se alimento o sistema, preencho o prontuário... às vezes ele é eletrônico, outras vezes, não [...]." (Médico USF 08)

"[...] toda hora tem que cadastrar, e olha que a população idosa não muda tanto, mas os dados parecem que somem. Se eu não tivesse meu caderninho, toda hora tinha que fazer de novo [...]." (Agente Comunitário de Saúde USF 1)

Diante desse panorama, o acompanhamento da população, por meio de recursos tecnológicos, torna-se um elemento essencial na busca pela atenção integral à saúde. Na APS, o uso dos sistemas de informação e do prontuário eletrônico oferece meios de avaliar a estrutura e monitorar indicadores de saúde que vão subsidiar a tomada de decisão centralizada nas reais necessidades dos indivíduos ${ }^{(22)}$.

Compete aos profissionais atuantes nas unidades de saúde da família o acompanhamento da população adscrita de sua área de abrangência como alicerce para uma assistência integral e equânime à saúde ${ }^{(2)}$. Nos relatos, observase que a equipe identifica como ações de acompanhamento do idoso as relacionadas puramente ao cuidado clínico:

\section{“[...] a gente tem o Dia do Idoso, tem o acompanhamento." (Recepcionista USF 2)}

"Ah, nós damos bastante atenção aos idosos: aferimos a pressão, medimos a glicemia." (Enfermeira USF 5) "É uma das coisas que funcionam aqui é o acompanhamento do idoso." (Enfermeira USF 9)

"A gente acompanha muito o idoso. A gente se preocupa muito com as feridas, com as medicações." (Enfermeira USF 10)

AAPS, como porta de entrada do usuário ao sistema de saúde, precisa fornecer acesso universal e igualitário, baseado na avaliação da gravidade do risco individual e coletivo e no critério cronológico, observadas as inúmeras especificidades previstas para pessoas com proteção especial, como a população idosa(6). Duas unidades manifestaram realizar atividade em grupo e citaram como ação a caminhada. Há, nas falas, uma fragmentação do cuidado quando os profissionais relatam que têm o "dia todo" de acompanhamento do idoso, o "dia do cronograma", além do enfoque na questão clínica, expressa na preocupação com "as feridas", "medicações", "aferição de pressão" e "glicemia", reforçando a cultura biomédica.

É urgente, no cenário contemporâneo, que se construam alternativas para uma atenção integral à saúde do idoso, na complexidade da realidade que a circunscreve, e que o foco seja o despreparo dos serviços de saúde, em especial no campo da APS, para lidar de forma adequada com essa questão. No âmbito do paradigma da funcionalidade, da atenção domiciliar e da formação profissional, percebe-se que os idosos estão sendo vistos em especialidades clínicas ou grupos de cuidados $^{(23)}$.

A efetivação da integralidade é permeada por inúmeros desafios, especialmente pelo forte poder do modelo biomédico e da produção ambulatorial, que ainda prevalecem no sistema de saúde ${ }^{(23)}$, de modo que poucos têm sido os movimentos que conseguem trazer à luz articulações de ações preventivas, curativas e de promoção à saúde com uma visão biopsicossocial articulada a um olhar ampliado e holístico para o indivíduo ${ }^{(24)}$. Os principais fatores que interferem na qualidade do atendimento, bem como na atenção integral ao idoso, são a demanda, a escassez de tempo e de profissionais, e a cobrança no alcance de metas ${ }^{(25)}$.

$A A M A Q-A B$ ressalta que a atenção integral à saúde do idoso pressupõe a realização de atendimento domiciliar aos idosos, especialmente daqueles impossibilitados de se locomover. Entende-se por atenção domiciliária a idosos todo o conjunto de ações realizadas por uma equipe multidisciplinar no domicílio do idoso e sua família ${ }^{(10)}$. 
No atual estudo, percebeu-se que as equipes da $A B$ realizam visitas domiciliares cotidianamente e as avaliam de forma satisfatória:

\section{"[...] Fazemos mais que uma visita por mês para os nossos idosos." (Enfermeira USF 4) \\ "É, acho que mais de $90 \%$ das visitas domiciliares são para os idosos." (Médico USF 4) \\ "[...] Realizamos visitas [...] aqueles que não podem vir, a família pede para ir lá no domicílio, a enfermeira e a médica vão." (Agente Comunitário de Saúde USF 5) \\ "Conseguimos monitorar as visitas, é satisfatório." (Médico USF 8)}

A visita domiciliar deve ser planejada com vistas ao atendimento de todas as necessidades de saúde do idoso e objetiva o acompanhamento dele e de seu familiar/cuidador, constituindo-se como um momento único na prestação de cuidado aos usuários, aproveitando o momento para prestar um atendimento que enfatize a autonomia e habilidades funcionais dentro de seu próprio ambiente. Assim, para além da realização das visitas, necessita-se de um planejamento a fim de responder às demandas sociais e de saúde da população atendida(26).

\section{Desafios na implementação da atenção qualificada ao idoso:}

O avançar da idade traz consigo transformações que devem ser foco no atendimento diferenciado, tais como alterações físicas, psicológicas e sociais. Além disso, no envelhecimento, pode haver diminuição da capacidade funcional, surgimento de doenças crônicas e degenerativas, dificuldades na realização das atividades básicas e instrumentais de vida diária, o que diminui a autonomia e o autocuidado. Isto requer da equipe de APS presença na rotina desse idoso ${ }^{(27,28)}$ e articulação com a rede de atenção e de cuidadores para promoção da autonomia e do autocuidado ${ }^{(29)}$.

Nos relatos abaixo, evidencia-se alguns aspectos que podem ser entendidos como desafios para a implementação da atenção qualificada ao idoso, dentre os quais: a capacitação de cuidadores, a atenção integral em saúde bucal e as práticas de educação em saúde.

$\mathrm{Na} A M A Q-A B^{(12)}$, alguns itens apontam para a realização de intervenções da APS com as famílias para identificação e capacitação dos cuidadores, visando à prestação de assistência domiciliária adequada. Nesta pesquisa, as equipes identificam os cuidadores, no entanto as atividades de capacitação não são realizadas:

“[...] identificar cuidadores sim, agora capacitar não. Já até intervimos em algumas coisas em relação aos cuidadores, mas capacitação não [...]." (Médico USF 6)

"capacitar cuidadores também não tem." (Agente Comunitário de Saúde USF 9)

Os profissionais de ensino superior são os mais indicados para orientar os cuidadores de idosos, especialmente no que diz respeito a tarefas que poderiam auxiliar na recuperação ou manutenção da qualidade de vida desses idosos, como: a prestação de apoio emocional e na convivência social; o auxílio e acompanhamento na realização de rotinas de higiene pessoal e ambiental; auxílio com nutrição, cuidados de saúde preventivos, administração de medicamentos e outros procedimentos de saúde; o auxílio e acompanhamento na mobilidade em atividades educativas, culturais, recreativas e de lazer ${ }^{(10)}$.

$\mathrm{Na}$ literatura, diferencia-se o cuidador formal do informal, sendo aquele um indivíduo que é remunerado para exercer a tarefa de cuidar e este um familiar, vizinho ou amigo que assume os cuidados voluntariamente ${ }^{(29)}$.

Na maioria das vezes, os cuidadores dos idosos fragilizados e/ou doentes são os próprios familiares. Logo, a devida rede de suporte e apoio é essencial. Pelo fato dos cuidadores nem sempre serem capacitados para prestação de cuidados, eles têm procurado cada vez mais a equipe de saúde para receber orientações. Nesse sentido, o processo de educação, orientação e capacitação dos cuidadores deve estar presente nos serviços de APS, e as informações sobre os cuidados adequados devem ser disseminadas e trabalhadas como práxis ${ }^{(30,31)}$.

O Ministério da Saúde afirma que o planejamento programado para a manutenção da saúde dos cuidadores e das famílias dos idosos necessitados tem sido frequentemente negligenciado e que a institucionalização ocorre, muitas vezes, em decorrência da inexistência de serviços suficientes capazes de assistir às necessidades tanto do idoso quanto de seu familiar/cuidador ${ }^{(31)}$.

A cobertura da assistência bucal para a atenção integral à saúde do idoso pressupõe a realização de exame da cavidade bucal ${ }^{(10)}$. No presente estudo, percebe-se que as equipes avaliaram a assistência bucal ofertada aos idosos como insatisfatória. 
"Nem todas as vezes faz o exame da cavidade oral." (Médica USF 8)

"Ah, na parte do dentista não funciona [...]." (Agente Comunitário de Saúde USF 10)

"Na saúde bucal é mais sintomáticos, é muito difícil. Eles não veem para todos. Consulta marcada não funciona muito [...]." (Cirurgião-dentista USF 10)

"Só queria trabalhar o programa, valorizar a consulta, porque eles só querem a consulta quando já estão sintomáticos." (Cirurgião-dentista USF 10)

Durante as reuniões, não se pontuou trabalhos específicos ou de grupo para a prevenção e promoção em saúde bucal. A saúde bucal dos idosos é comprometida por uma série de fatores que os tornam susceptíveis a diversas alterações e lesões de mucosa, especialmente à presença de condições patológicas próprias ao envelhecimento, por isso a promoção da saúde bucal na terceira idade é fundamental ${ }^{(32)}$.

Diante dessa realidade, a equipe de saúde bucal da $A B$ deve executar e priorizar ações e avaliações da saúde bucal desse público, dentre elas: realização de consulta e avaliação do quadro clínico; diagnóstico e tratamento restaurador; solicitação de exames complementares; pequenas cirurgias; prescrição de medicamentos; realização de assistência domiciliar; orientação à pessoa idosa, aos familiares e/ou cuidador sobre a importância da higienização da boca e da prótese; e encaminhar, quando necessário, para referências de média e alta complexidade em saúde bucal(31).

Com o aumento da expectativa de vida, a saúde bucal do idoso torna-se necessária no SUS. Nesse sentido, o profissional cirurgião-dentista deve atuar em conjunto com a equipe na busca por ações intersetoriais de promoção de saúde e todos devem trabalhar almejando o alcance pela qualidade de vida e autoestima do idoso ${ }^{(33)}$.

Atividades desenvolvidas nas unidades de saúde, como as práticas de educação em saúde, são fundamentais para a ampliação e disseminação do conhecimento e conferem protagonismo ao autocuidado ${ }^{(34)}$. Evidencia-se nas falas dos profissionais a desvalorização das práticas de educação em saúde voltadas ao idoso, como se observa nos trechos abaixo:

"Querida, já vi que terá que fechar a unidade para fazer só educação em saúde (risos) e grupos [...]." (Médica USF 1)

"[...] nós não vamos conseguir nunca chegar nisso. Não vamos conseguir nunca fazer prevenção, aqui atendemos demanda que chega." (Técnica de Enfermagem USF 1)

Na fala do profissional médico há uma dissociação da educação em saúde com as outras práticas em saúde. A educação em saúde é instrumental da APS, no entanto percebe-se que há desvalorização dessas ações pelos próprios profissionais que atuam nesse âmbito( ${ }^{(3)}$. No caso apresentado, é necessário um investimento para reconhecer que espaços intrínsecos na prática profissional, tais como o acolhimento, as consultas e as visitas domiciliares, são espaços de educação em saúde, e não necessariamente o grupo deve somente fomentar essa ação.

No século passado, essas práticas constituíram-se em uma das principais formas de promoção à saúde, viabilizaram mudanças significativas na saúde pública e promoveram avanços na medicina curativa e preventiva, na contribuição da longevidade, na redução das taxas de mortalidade e melhor qualidade de vida no planeta ${ }^{(36)}$. As estratégias de educação em saúde têm influência significativa sobre a saúde e o estilo de vida de pessoas. No caso do idoso, ela pode ser a principal alternativa para promover saúde ${ }^{(37)}$.

Nesse sentido, um estudo realizado a fim de analisar o impacto das ações de promoção e educação em saúde, na busca pela qualidade de vida do idoso, concluiu que a educação em saúde se constitui numa poderosa ferramenta de promoção da saúde e garantia da autonomia e autocuidado do idoso(38).

A atenção especial deve ser dispensada aos recursos humanos. No que se refere à capacitação em atenção à saúde do idoso, torna-se necessário investimento de gestores da Secretaria Municipal de Saúde em programas de capacitação profissional direcionado ao cuidado integral a pessoa idosa ${ }^{(39)}$.

As limitações desta investigação dizem respeito à inclusão de unidades avaliadas pelo PMAQ. Se, por um lado, pode denotar limite, por outro, pode representar determinada caracterização dessas unidades. Quanto a não participação de usuários e dos cuidadores de idosos, embora também seja uma restrição nesse momento, a opção foi por privilegiar profissionais cuja responsabilidade pelo cuidado é diferenciada.

É importante ressaltar que os participantes do presente estudo não abordaram questões referentes à realização de monitoramento da cobertura vacinal e intervenções de detecção precoce das demências. Perceberam-se, no contexto do estudo, a fragmentação e a desarticulação como importantes problemas na assistência em saúde 
da atenção básica ${ }^{(40)}$, uma vez que o trabalho realizado sofre influência do modelo biomédico. É necessária uma remodelagem do serviço e a construção de um novo modelo assistencial, alicerçado nos princípios do SUS, buscando a manutenção da funcionalidade do idoso e sua qualidade de vida, sendo a promoção da saúde e o cuidado integral os focos principais da assistência ${ }^{(23,25)}$.

\section{CONSIDERAÇÕES FINAIS}

O presente estudo evidenciou que o cuidado ao idoso nas Unidades de Saúde da Família ocorre de maneira fragmentada, reforça a cultura biomédica e perpassa sobremaneira pela clínica realizada rotineiramente nas unidades e/ou no espaço domiciliar, caracterizando-se por um conjunto de ações curativas em detrimento das preventivas.

No contexto desta investigação, as ações de promoção da saúde mostraram-se insuficientes para garantir a atenção integral, uma vez que se encontram desarticuladas e subutilizadas pelos profissionais, sendo identificadas fragilidades nas práticas de educação em saúde, a não utilização da Caderneta de Saúde do Idoso, falta de capacitação dos cuidadores, pouco acompanhamento dos usuários portadores de condições crônicas que requerem percorrer os níveis de atenção à saúde, além de baixo uso do programa de saúde bucal e, em algumas unidades, a ausência.

\section{AGRADECIMENTOS E CONFLITOS DE INTERESSE}

Agradecimento à Secretaria Municipal de Saúde de Tangará da Serra, Mato Grosso.

Não existem conflitos de interesse.

\section{CONTRIBUIÇÕES}

Juliana Fernandes Cabral contribuiu com a elaboração e o delineamento do estudo; Jeniffer Fernanda Gonçalves da Silva, Josué Souza Gleriano e Priscila Balderrama contribuíram com a aquisição, análise e interpretação dos dados; Angélica Pereira Borges e Ageo Mário Cândido da Silva contribuíram com a redação e/ou revisão do manuscrito.

\section{REFERÊNCIAS}

1. Brasil. Conselho Nacional de Secretários de Saúde. SUS 20 anos [Internet]. Brasília: CONASS; 2009. [acesso em 2018 Out 27]. Disponível em: http://www.conass.org.br/bibliotecav3/pdfs/sus20anosfinal.pdf

2. Brasil. Ministério da Saúde. Portaria n. 2.436, de 21 de setembro de 2017. Política Nacional de Atenção Básica [Internet]. Diário Oficial da União; Brasília; 22 Set 2017; [acesso em 2018 Abr 24]. Disponível em: http://bvsms.saude.gov.br/bvs/saudelegis/gm/2017/prt2436_22_09_2017.html

3. Mendes EV. Entrevista: a abordagem das condições crônicas pelo Sistema Único de Saúde. Ciênc Saúde Colet. [Internet]. 2018 [acesso em 2018 Jul 5];23(2):431-5. Disponível em: http://www.scielo.br/pdf/csc/ v23n2/1413-8123-csc-23-02-0431.pdf

4. Furtado JP, Vieira-da-Silva LM. A Avaliação de programas e serviços de saúde no Brasil enquanto espaço de saberes e práticas. Cad Saúde Pública [Internet]. 2014 [acesso em 2018 Jun 25];30(12):2643-55. Disponível em: http://www.scielo.br/pdf/csp/v30n12/0102-311X-csp-30-12-02643.pdf

5. Chaves LDP, Jesus BJ, Ferreira JBB, Balderrama P, Tanaka OY. Avaliação de resultados da atenção aos agravos cardiovasculares como traçador do princípio de integralidade. Saúde Soc [Internet]. 2015 [acesso em 2018 Abr 10];24(2):568-77. Disponível em: http://www.scielo.br/pdf/sausoc/v24n2/0104-1290sausoc-24-02-00568.pdf

6. Brasil. Decreto n. 7.508, de 28 de junho de 2011. Regulamenta a Lei no 8.080, de 19 de setembro de 1990, para dispor sobre a organização do Sistema Único de Saúde - SUS, o planejamento da saúde, a assistência à saúde e a articulação interfederativa, e dá outras providências [Internet]. Diário Oficial da União; Brasília; 29 Jul 2011; [acesso em 2018 Mar 10]. Disponível em: http://www.planalto.gov.br/ccivil_03/_Ato2011-2014/2011/ Decreto/D7508.htm

7. Gleriano JS, Lucietto GC, Reis JB, Teixeira VM, Chaves LDP. A percepção de enfermeiros da estratégia saúde da família sobre o conceito e prática da avaliação. Rev Cuid [Internet]. 2017 [acesso em 2018 Jul 13];11(2):248-56. Disponível em: http://www.webfipa.net/facfipa/ner/sumarios/cuidarte/2017v2/248.pdf 
8. Brasil. Ministério da Saúde. Saúde mais perto de você - Acesso e qualidade. Programa Nacional de Melhoria do Acesso e da Qualidade da Atenção Básica (PMAQ): manual instrutivo [Internet]. Brasília: Ministério da Saúde; 2012 [acesso em 2018 Mar 15]. Disponível em: http://189.28.128.100/dab/docs/publicacoes/geral/ manual_instrutivo_pmaq_site.pdf

9. Dilélio AS, Tomasi E, Thumé E, Silveira DS, Siqueira FCV, Piccini RX. et al. Padrões de utilização de atendimento médico-ambulatorial no Brasil entre usuários do Sistema Único de Saúde, da saúde suplementar e de serviços privados. Cad Saúde Pública [Internet]. 2014 [acesso em 2018 Fev 9];30(12):2594-606.

Disponível em: http://www.scielo.br/pdf/csp/v30n12/0102-311X-csp-30-12-02594.pdf

10. (Br). Ministério da Saúde. Autoavaliação para melhoria do acesso e da qualidade na Atenção Básica - AMAQ [Internet]. 2a ed. Brasília: Ministério da Saúde; 2016 [acesso em 2018 Abr 15]. Disponível em: http://bvsms. saude.gov.br/bvs/publicacoes/autoavaliacao_melhoria_acesso_qualidade_amaq_2ed.pdf

11. Governo do Brasil. Brasil fará parte da pesquisa internacional sobre o idoso [Internet]. 2017 [acesso em 2018 Out 26]. Disponível em: http://www.brasil.gov.br/noticias/saude/2012/10/brasil-fara-parte-de-pesquisainternacional-sobre-idoso

12. Brasil. Ministério da Saúde. Autoavaliação para melhoria do acesso e da qualidade na Atenção Básica - AMAQ [Internet]. Brasília: Ministério da Saúde; 2017 [acesso em 2018 Out 25]. Disponível em: http://189.28.128.100/dab/docs/portaldab/publicacoes/amaq_2017.pdf

13. Morgan DL. Focus groups as qualitative research. Qualitative Research Methods Series. London: Sage Publications; 1997.

14. Weber F. A Entrevista, a pesquisa e o íntimo, ou: por que censurar seu diário de campo? Rev Horiz Antropol [Internet]. 2009 [acesso em 2018 Out 26];15(32):157-70. Disponível em: http://www.scielo.br/pdf/ha/v15n32/ v15n32a07.pdf

15. Campos RTO, Furtado JP. Narrativas: utilização na pesquisa qualitativa em saúde. Rev Saúde Pública [Internet]. 2008 [acesso em 2018 Out 26];42(6):1090-6. Disponível em http://www.scielo.br/pdf/ rsp/2008nahead/7066.pdf

16. Bardin L. Análise de conteúdo. Coimbra: Ed. 70; 2011.

17. Fontanella BJB, Luchesi BM, Saidel MGB, Ricas J, Turato ER, Melo DG. Amostragem em pesquisas qualitativas: proposta de procedimentos para constatar saturação teórica. Cad Saúde Pública [Internet]. 2011 [acesso em 2018 Out 26];27(2):388-94. Disponível em: http://www.scielo.br/pdf/csp/v27n2/20.pdf

18. SÁ CMCP. Caderneta de saúde da pessoa idosa no olhar dos profissionais da estratégia de saúde da família [Dissertação]. João Pessoa: Universidade Federal de Paraíba; 2016 [acesso em 2018 Fev 15]. Disponível em: https://repositorio.ufpb.br/jspui/bitstream/tede/8732/2/arquivototal.pdf

19. (Br). Ministério da Saúde. Caderneta de saúde da pessoa idosa [Internet]. 4a ed. Brasília: Ministério da Saúde. 2017 [acesso em 2018 Fev 15]. Disponível em: http://portalarquivos2.saude.gov.br/images/pdf/2017/ setembro/27/CADERNETA-PESSOA-IDOSA-2017-Capa-miolo.pdf

20. Assembleia Legislativa do Estado de Mato Grosso. Manifestação de interesse na aquisição da Caderneta de Saúde do Idoso [Internet]. 2017 [acesso em 2017 dez 17]. Disponível em: http://www.al.mt.gov.br/storage/ webdisco/cp/20150806085417146000.pdf

21. Bousquat A, Giovanella L, Fausto MCR, Fusaro ER, Mendonça MHM, Gagno J; et al. Tipologia da estrutura das unidades básicas de saúde brasileiras: os 5 R. Cad Saúde Pública. [Internet]. 2017 [acesso em 2018 Out 27];33(8):e00037316. Disponível em: http://www.scielo.br/pdf/csp/v33n8/1678-4464-csp-33-08-e00037316. pdf

22. Soranz D, Pinto LF, Camacho LAB. Analysis of the attributes of primary health care using the electronic medical records in the city of Rio de Janeiro. Ciênc Saúde Colet [Internet]. 2017 [acesso em 2018 Out 27];22(3):819-30. Disponível em: http://www.scielo.br/pdf/csc/v22n3/en_1413-8123-csc-22-03-0819.pdf

23. Medeiros KKAS, Pinto EP Jr, Bousquat A, Medina MG. O desafio da integralidade no cuidado ao idoso, no âmbito da Atenção Primária em Saúde. Saúde Debate [Internet]. 2017 [acesso em 2018 Jul 1];41(3):288-95. Disponível em: http://www.scielo.br/pdf/sdeb/v41nspe3/0103-1104-sdeb-41-spe3-0288.pdf 
24. Carvalho G. A saúde pública no Brasil. Estud Av [Internet]. 2013 [acesso em 2018 Fev 16];27(78):7-26. Disponível em: http://www.scielo.br/scielo.php?script=sci_arttext\&pid=S0103-40142013000200002

25. Coutinho AT, Popim RC, Carregã K, Spiri WC. Integralidade do cuidado com o idoso na estratégia de saúde da família: visão da equipe. Esc Anna Nery Rev Enferm [Internet]. 2013 [acesso em 2018 Fev 26];17(4):62837. Disponível em: http://www.scielo.br/pdf/ean/v17n4/1414-8145-ean-17-04-0628.pdf

26. Mello DRB, Apratto PC Jr, César TPO, Souza D, Miranda D, Freitas G, et al. Fatores de resiliência no envelhecimento verificados na visita domiciliar: relato de uma experiência na atenção básica. REINPEC [Internet]. 2016 [acesso em 2017 Dez 15];2(2):30-44. Disponível em: http://reinpec.srvroot.com:8686/reinpec/ index.php/reinpec/article/view/200/70

27. Barbosa AS, Andrade GCL, Pereira CO, Falcão IV. A interdisciplinaridade vivenciada em um grupo de idosos de uma unidade de saúde da família do Recife. Rev APS [Internet]. 2016 [acesso em 2018 Jan 10];19(2):31520. Disponível em: https://aps.ufjf.emnuvens.com.br/aps/article/view/2247/978

28. Ferreira LV, Silva MCM, Castro EAB, Friedrich DBC. Busca do autocuidado por idosos na rede de atenção à saúde. Rev Contexto Saúde [Internet]. 2017 [acesso em 2018 Jan 10];17(32):46-54. Disponível em: https:// www.revistas.unijui.edu.br/index.php/contextoesaude/article/view/5984

29. Lethin C, Leino-Kilpi H, Roe B, Soto MM, Saks K, Stephan A, et al. Formal support for informal caregivers to older persons with dementia through the course of the disease: an exploratory, cross-sectional study. BMC Geriatr [Internet]. 2016 [acesso em 2018 Jan 10];16(32). Disponível em: https://bmcgeriatr.biomedcentral. com/articles/10.1186/s12877-016-0210-9

30. Anício VA. Cuidando de idosos: um enfoque na capacitação do cuidador [Trabalho de Conclusão de Curso]. Conselheiro Lafaiete (MG): Universidade Federal de Minas Gerais. 2013 [acesso em 2018 Jan 15]. Disponível em: https://www.nescon.medicina.ufmg.br/biblioteca/imagem/4167.pdf

31. (Br). Ministério da Saúde. Envelhecimento e saúde da pessoa idosa [Internet]. Brasília: DF; 2006 [acesso em 2017 Dez 20]. Disponível em: http://dab.saude.gov.br/portaldab/biblioteca.php?conteudo=publicacoes/cab19

32. Sales MVG, Fernandes JA Neto, Catão MHCV. Condições de saúde bucal do idoso no Brasil: uma revisão de literatura. Arch Health Invest [Internet]. 2017 [acesso em 2018 Jan 10];6(3):120-4. Disponível em: http://www. archhealthinvestigation.com.br/ArcHI/article/view/1918/pdf

33. Agudelo-Suárez AA, López-Vergel F, Alzate-Urrea S, López-Orozco C, Espinosa-Herrera E, Posada-López A; et al. Salud bucal y género en relación a la población adulta mayor atendida en la red hospitalaria pública de Medellín: los puntos de vista del personal de salud. Univ Salud [Internet]. 2016 [acesso em $2018 \mathrm{Jul}$ 1];18(1):58-68. Disponível em: http://www.scielo.org.co/pdf/reus/v18n1/v18n1a07.pdf

34. Soares CF, Heidemann ITSB. Promoção da saúde e prevenção da lesão por pressão: expectativas do enfermeiro da atenção primária. Texto \& Contexto Enferm [Internet]. 2018 [acesso em 2018 Jan 9];27(2):e1630016. Disponível em: http://www.scielo.br/pdf/tce/v27n2/0104-0707-tce-27-02-e1630016.pdf

35. Santili PGJ, Tonhom SFR, Marin MJS. Educação em saúde: algumas reflexões sobre sua implementação pelas equipes da estratégia saúde da família. Rev Bras Promoç Saúde [Internet]. 2016 [acesso em 2018 Jan 9];29(Supl):102-10. Disponível em: http://periodicos.unifor.br/RBPS/article/view/6411/5218

36. Ochoa AMG. Educación y salud: dos campos de intervención, un interés común. Rev Colomb Educ [Internet]. 2013 [acesso em 2018 Jan 10];65:123-52. Disponível em: http://www.scielo.org.co/pdf/rcde/n65/n65a07.pdf

37. Mallmann DG, Galindo NM Neto, Sousa JC, Vasconcelos EMR. Educação em saúde como principal alternativa para promover a saúde do idoso. Ciênc Saúde Colet [Internet]. 2015 [acesso em $2018 \mathrm{Fev}$ 16];20(6):1763-72. Disponível em: http://www.scielo.br/pdf/csc/v20n6/1413-8123-csc-20-06-1763.pdf

38. Janini JP, Bessler D, Vargas AB. Educação em saúde e promoção da saúde: impacto na qualidade de vida do idoso. Saúde Debate [Internet]. 2015 [acesso em 2018 Jul 1];39(105):480-90. Disponível em: http://www. scielo.br/pdf/sdeb/v39n105/0103-1104-sdeb-39-105-00480.pdf

39. Lima ES, Oliveira APP, Esteves AVF. Cuidar do idoso na atenção primária de saúde: dificuldades enfrentadas pelo enfermeiro. REAS [Internet]. 2018 [acesso em 2018 Jul 12];10(1):1395-403. Disponível em: https://www. acervosaude.com.br/doc/REAS118.pdf 
40. Campos GWS. Subjetividade e administração de pessoal: considerações sobre modos de gerenciar o trabalho em equipes de saúde. In: Merhy EE, Onocko R, organizadores. Agir em saúde: um desafio para o público. São Paulo: Hucitec; 1997. p. 229-66.

\section{Endereço do primeiro autor:}

Juliana Fernandes Cabral

Universidade do Estado de Mato Grosso - UNEMAT

Rod. MT 358, KM 07, Bloco de Enfermagem

Bairro: Jardim Aeroporto

CEP: 78.300-000 - Tangará da Serra - MT - Brasil

E-mail: julianacabral@unemat.br

\section{Endereço para correspondência:}

Josué Souza Gleriano

Universidade do Estado de Mato Grosso - UNEMAT

Rod. MT 358, KM 07, Bloco de Enfermagem

Bairro: Jardim Aeroporto

CEP: 78.300-000 - Tangará da Serra - MT - Brasil

E-mail: josuegleriano@unemat.br

Como citar: Cabral JF, da Silva JFG, Gleriano JS, Balderrama P, Borges AP, da Silva AMC. Avaliação da atenção integral à saúde do idoso na percepção de profissionais. Rev Bras Promoç Saúde. 2019;32:8367. 